\title{
EPIMORPHICALLY CLOSED PERMUTATIVE VARIETIES
}

BY

\author{
N. M. KHAN
}

\begin{abstract}
We show that for semigroups all permutation identities are preserved under epis and that all subvarieties of the permutative variety defined by any permutation identity

$$
x_{1} x_{2} \cdots x_{n}=x_{i_{1}} x_{i_{2}} \cdots x_{i_{n}},
$$

with $n \geqslant 3$ and such that $i_{n} \neq n$ or $i_{1} \neq 1$, are closed under epis. Finally we find some sufficient conditions that an identity be preserved under epis in conjunction with any nontrivial permutation identity.
\end{abstract}

1. Introduction and summary. We establish that for semigroups all permutation identities are preserved under epis. A stronger result for commutativity has long been known, namely that the semigroup dominion of a commutative semigroup is also commutative; we show by a counterexample due to P. M. Higgins that this stronger result is false for each (nontrivial) permutation identity other than commutativity. Next we show that all subvarieties of the permutative variety defined by any permutation identity

$$
x_{1} x_{2} \cdots x_{n}=x_{i_{1}} x_{i_{2}} \cdots x_{i_{n}},
$$

with $n \geqslant 3$ and such that $i_{n} \neq\left[i_{1} \neq 1\right]$, are closed under epis, thus generalizing Theorem 4.1 of the author [10] which states that all commutative varieties are closed under epis. Finally we find some sufficient conditions that an identity be preserved under epis in conjunction with any nontrivial permutation identity.

2. Preliminaries. Let $U, S$ be semigroups with $U$ a subsemigroup of $S$. We say that $U$ dominates an element $d$ of $S$ if for every semigroup $T$ and for all homomorphisms $\beta, \gamma: S \rightarrow T, u \beta=u \gamma$ for all $u \in U$ implies $d \beta=d \gamma$. The set of all elements of $S$ dominated by $U$ is called the dominion of $U$ in $S$, and we denote it by $\operatorname{Dom}_{S}(U)$. It can be easily verified that $\operatorname{Dom}_{S}(U)$ is a subsemigroup of $S$ containing $U$. Following Howie and Isbell [8], we call a semigroup $U$ saturated if $\operatorname{Dom}_{S}(U) \neq S$ for every properly contained semigroup $S$.

A morphism $\alpha: A \rightarrow B$ in the category $\mathscr{C}$ of semigroups is called an epimorphism (epi for short) if for all $C \in \mathscr{C}$ and for all morphisms $\beta, \gamma: B \rightarrow C, \alpha \beta=\alpha \gamma$ implies $\beta=\gamma$. It can be easily verified that a morphism $\alpha: S \rightarrow T$ is epi if and only if the inclusion map $i$ : $S \alpha \rightarrow T$ is epi, and the inclusion map $i: U \rightarrow S$ from any

Received by the editors June 30, 1983 and, in revised form, November 15, 1983.

1980 Mathematics Subject Classification. Primary 20M07.

Key words and phrases. Semigroup, variety, epimorphism, dominion.

C1985 American Mathematical Society $0002-9947 / 85 \$ 1.00+\$ .25$ per page 
subsemigroup $U$ of $S$ is epi if and only if $\operatorname{Dom}_{S}(U)=S$. In such a case $S$ will be called an epimorphic extension of $U$.

A most useful characterization of semigroup dominions is provided by Isbell's Zigzag Theorem.

Result 1 [9, Theorem 2.3 or 7, Theorem VII.2.13]. Let $U$ be a subsemigroup of any semigroup $S$, and let $d$ be any element of $S$. Then $d \in \operatorname{Dom}_{S}(U)$ if and only if either $d \in U$ or there are elements $a_{0}, a_{1}, a_{2}, \ldots, a_{2 m} \in U, t_{1}, t_{2}, \ldots, t_{m}, y_{1}, y_{2}, \ldots, y_{m}$ $\in S$ such that

$$
\begin{aligned}
& d=a_{0} t_{1}, \quad a_{0}=y_{1} a_{1}, \\
& y_{i} a_{2 i}=y_{i+1} a_{2 i+1}, \quad a_{2 i-1} t_{i}=a_{2 i} t_{i+1} \quad(i=1,2, \ldots, m-1), \\
& a_{2 m-1} t_{m}=a_{2 m}, \quad y_{m} a_{2 m}=d .
\end{aligned}
$$

These equations are called a zigzag of length $m$ over $U$ with value $d$ and with spine $a_{0}$, $a_{1}, a_{2}, \ldots, a_{2 m}$.

An identity of the form of equation (1) for some permutation $i$ of the set $\{1,2, \ldots, n\}$ is called a permutation identity. The permutation identity (1) is said to be nontrivial if the permutation $i$ is different from the identity permutation.

RESULT 2 [11, Result 3]. Let $U$ and $S$ be any semigroups with $U$ a subsemigroup of $S$. For any $d \in \operatorname{Dom}_{S}(U) \backslash U$, if (2) is a zigzag of shortest possible length $m$ over $U$ with value $d$, then $t_{j}, y_{j} \in S \backslash U$ for $j=1,2, \ldots, m$.

RESUlT 3 [11, Proposition 3.1]. Let $S$ be any semigroup satisfying the identity (1) with $n \geqslant 3$.

(i) For each $j \in\{2,3, \ldots, n\}$ such that $x_{j-1} x_{j}$ is not a subword of $x_{i_{1}} x_{i_{2}} \cdots x_{i_{n}}, S$ also satisfies the permutation identity

$$
x_{1} x_{2} \cdots x_{j-1} x y x_{j} \cdots x_{n}=x_{1} x_{2} \cdots x_{j-1} y x x_{j} \cdots x_{n} .
$$

(ii) If $x_{1} \neq x_{i_{1}}$, then $S$ also satisfies the permutation identity

$$
x y x_{1} x_{2} \cdots x_{n}=y x x_{1} x_{2} \cdots x_{n} \text {. }
$$

In the following results, let $U$ and $S$ be any semigroups with $U$ a subsemigroup of $S$ and such that $\operatorname{Dom}_{S}(U)=S$.

RESULT 4 [11, Result 4]. If $d \in S \backslash U$, then for any positive integer $k$, there exist $a_{1}, a_{2}, \ldots, a_{k} \in U$ and $d_{k} \in S \backslash U$ such that $d=a_{1} a_{2} \cdots a_{k} d_{k}$.

RESUlT 5 [11, Corollary 4.4]. Let $U$ satisfy a permutation identity (1) with $i_{n} \neq n$. Then, for each positive integer $k$,

$$
s x_{1} x_{2} \cdots x_{k}=s x_{j_{1}} x_{j_{2}} \cdots x_{j_{k}}
$$

for all $x_{1}, x_{2}, \ldots, x_{k} \in S, s \in S \backslash U$, and for any permutation $j$ of the set $\{1,2, \ldots, k\}$.

RESUlT 6 [11, Corollary 4.2]. If $U$ satisfies a nontrivial permutation identity, then for each positive integer $k$,

$$
s x_{1} x_{2} \cdots x_{k} t=s x_{j_{1}} x_{j_{2}} \cdots x_{j_{k}} t
$$

for all $s, t \in S \backslash U, x_{1}, x_{2}, \ldots, x_{k} \in S$, and for any permutation $j$ of the set $\{1,2, \ldots, k\}$.

The notations and conventions of Clifford and Preston [3] and Howie [7] will be used throughout without explicit mention. 
The general question of which varieties are closed under epis has been studied in semigroup theory, ring theory and elsewhere [2]. For example, in [4] Gardner has shown that certain identities weaker than commutativity are not preserved under epis of rings although the variety of commutative rings is closed under epis [1]. P. M. Higgins [5] has shown that identities for which both sides contain a repeated variable are not preserved under epis of semigroups. In showing that all varieties of commutative semigroups are closed under epis [10], the author has generalized the classic result of Isbell [9, Corollary 2.5] that commutativity is preserved under epis. However, finding a complete determination of all identities which are preserved under epis of semigroups still remains an open problem.

3. Epimorphisms of semigroups and permutation identities. An identity $u=v$ is said to be preserved under epis if for all semigroups $U$ and $S$ with $U$ a subsemigroup of $S$ and such that $\operatorname{Dom}_{S}(U)=S, U$ satisfying $u=v$ implies $S$ satisfies $u=v$.

THEOREM 3.1. All permutation identities are preserved under epis.

Proof. Let equation (1) be any permutation identity with $n \geqslant 3$. Without loss we can assume that (1) is nontrivial. Take any semigroup $U$ satisfying (1), and any semigroup $S$ containing $U$ properly and such that $\operatorname{Dom}_{S}(U)=S$. We shall show that $S$ also satisfies (1).

For $k=1,2, \ldots, n$, consider the word $x_{i_{1}} x_{i_{2}} \cdots x_{i_{k}}$ of length $k$. We shall prove the theorem by induction on the length of these words, assuming that the remaining elements $x_{i_{k+1}}, \ldots, x_{i_{n}} \in U$.

First for $k=1$, that is, when $x_{i_{1}} \in S$, and $x_{i_{2}}, \ldots, x_{i_{n}} \in U$, we wish to show that equation (1) holds. When $x_{i_{1}} \in U$, (1) holds so we assume that $x_{i_{1}} \in S \backslash U$. By Result 1, we may let (2) be a zigzag of shortest possible length $m$ over $U$ with value $x_{i_{1}}$.

First we introduce some notation:

$$
\begin{aligned}
& w_{1}\left(x_{i_{1}}, x_{i_{2}}, \ldots, x_{i_{n}}\right)=x_{i_{1}} x_{i_{2}} \cdots x_{i_{n}}=u_{1}\left(x_{1}, x_{2}, \ldots, x_{n}\right), \\
& w_{2}\left(x_{i_{1}}, x_{i_{2}}, \ldots, x_{i_{n}}\right)=x_{1} x_{2} \cdots x_{n}=u_{2}\left(x_{1}, x_{2}, \ldots, x_{n}\right) .
\end{aligned}
$$

Case (i). $i_{1}=1$. Now

as required.

$$
\begin{aligned}
x_{i_{1}} x_{i_{2}} \cdots x_{i_{n}} & =y_{m} a_{2 m} x_{i_{2}} \cdots x_{i_{n}}=y_{m} w_{1}\left(a_{2 m}, x_{i_{2}}, \ldots, x_{i_{n}}\right) \\
& =y_{m} w_{2}\left(a_{2 m}, x_{i_{2}}, \ldots, x_{i_{n}}\right) \quad(\text { since } U \text { satisfies }(1)) \\
& =y_{m} a_{2 m} x_{2} \cdots x_{n}=x_{1} x_{2} \cdots x_{n},
\end{aligned}
$$

Case (ii). $1<i_{1}<n$. Now, putting $j=i_{1}$, we have

(4) $x_{i_{1}} x_{i_{2}} \cdots x_{i_{n}}=y_{m} a_{2 m} x_{i_{2}} \cdots x_{i_{n}}$ (from equations (2))

$$
\begin{aligned}
& =y_{m} w_{1}\left(a_{2 m}, x_{i_{2}}, \ldots, x_{i_{n}}\right) \\
& =y_{m} w_{2}\left(a_{2 m}, x_{i_{2}}, \ldots, x_{i_{n}}\right) \quad(\text { since } U \text { satisfies }(1)) \\
& =y_{m} x_{1} x_{2} \cdots x_{j-1} a_{2 m} x_{j+1} \cdots x_{n} \\
& =y_{m} x_{1} x_{2} \cdots x_{j-1} a_{2 m-1} t_{m} z \quad(\text { from equations (2), }
\end{aligned}
$$

where $\left.z=x_{j+1}, \ldots, x_{n}\right)$

(Continues) 


$$
\begin{array}{r}
=y_{m} x_{1} x_{2} \cdots x_{j-1} a_{2 m-1} b_{j+1}^{(m)} \cdots b_{n}^{(m)} t_{m}^{\prime} z \\
\quad \text { (by Result } 4, \text { for some } b_{j+1}^{(m)}, \ldots, b_{n}^{(m)} \in U, \\
\text { and } \left.t_{m}^{\prime} \in S \backslash U, \text { since } t_{m} \in S \backslash U\right) \\
=y_{m} u_{2}\left(x_{1}, x_{2}, \ldots, x_{j-1}, a_{2 m-1}, b_{j+1}^{(m)}, \ldots, b_{n}^{(m)}\right) t_{m}^{\prime} z \\
=y_{m} u_{1}\left(x_{1}, x_{2}, \ldots, x_{j-1}, a_{2 m-1}, b_{j+1}^{(m)}, \ldots, b_{n}^{(m)}\right) t_{m}^{\prime} z
\end{array}
$$

(since $U$ satisfies (1)).

Now $u_{1}\left(z_{1}, z_{2}, \ldots, z_{n}\right)$ begins with $z_{i_{1}}=z_{j}$, so the product (4) in $S$ contains $y_{m} a_{2 m-1}$ which equals $y_{m-1} a_{2 m-2}$ (from equations (2)). Thus the product (4) above equals

$$
\begin{aligned}
y_{m-1} & u_{1}\left(x_{1}, x_{2}, \ldots, x_{j-1}, a_{2 m-2}, b_{j+1}^{(m)}, \ldots, b_{n}^{(m)}\right) t_{m}^{\prime} z \\
& \left.=y_{m-1} u_{2}\left(x_{1}, x_{2}, \ldots, x_{j-1}, a_{2 m-2}, b_{j+1}^{(m)}, \ldots, b_{n}^{(m)}\right) t_{m}^{\prime} z \quad \text { (since } U \text { satisfies }(1)\right) \\
& =y_{m-1} x_{1} x_{2} \cdots x_{j-1} a_{2 m-2} b_{j+1}^{(m)} \cdots b_{n}^{(m)} t_{m}^{\prime} z \\
& =y_{m-1} x_{1} x_{2} \cdots x_{j-1} a_{2 m-2} t_{m} z \quad\left(\text { since } t_{m}=b_{j+1}^{(m)} \cdots b_{n}^{(m)} t_{m}^{\prime} z\right) \\
& =y_{m-1} x_{1} x_{2} \cdots x_{j-1} a_{2 m-3} t_{m-1} z \quad(\text { from equations }(2)) \\
& \vdots \\
& =y_{1} x_{1} x_{2} \cdots x_{j-1} a_{1} t_{1} z \\
& =y_{1} x_{1} x_{2} \cdots x_{j-1} a_{1} b_{j+1}^{(1)} \cdots b_{n}^{(1)} t_{1}^{\prime} z
\end{aligned}
$$

(by Result 4, for some

$$
\begin{aligned}
& \left.b_{j+1}^{(1)}, \ldots, b_{n}^{(1)} \in U, \text { and } t_{1}^{\prime} \in S \backslash U, \text { since } t_{1} \in S \backslash U\right) \\
= & y_{1} u_{2}\left(x_{1}, x_{2}, \ldots, x_{j-1}, a_{1}, b_{j+1}^{(1)}, \ldots, b_{n}^{(1)}\right) t_{1}^{\prime} z \\
= & y_{1} u_{1}\left(x_{1}, x_{2}, \ldots, x_{j-1}, a_{1}, b_{j+1}^{(1)}, \ldots, b_{n}^{(1)}\right) t_{1}^{\prime} z \quad(\text { since } U \text { satisfies }(1)) .
\end{aligned}
$$

Again as before, product (5) in $S$ contains $y_{1} \dot{a}_{1}$ which equals $a_{0}$ (from equations (2)). Thus the product (5) above equals

$$
\begin{aligned}
u_{1}\left(x_{1},\right. & \left.x_{2}, \ldots, x_{j-1}, a_{0}, b_{j+1}^{(1)}, \ldots, b_{n}^{(1)}\right) t_{1}^{\prime} z \\
& =u_{2}\left(x_{1}, x_{2}, \ldots, x_{j-1}, a_{0}, b_{j+1}^{(1)}, \ldots, b_{n}^{(1)}\right) t_{n}^{\prime} z \quad(\text { since } U \text { satisfies }(1)) \\
& =x_{1} x_{2} \cdots x_{j-1} a_{0} b_{j+1}^{(1)} \cdots b_{n}^{(1)} t_{1}^{\prime} z \\
& =x_{1} x_{2} \cdots x_{j-1} a_{0} t_{1} z \quad\left(\text { since } t_{1}=b_{j+1}^{(1)} \cdots b_{n}^{(1)} t_{1}^{\prime}\right) \\
& =x_{1} x_{2} \cdots x_{n} \quad\left(\text { since } a_{0} t_{1}=x_{i_{1}}=x_{j}, \text { and } z=x_{j+1} \cdots x_{n}\right)
\end{aligned}
$$

which proves the result for $k=1$ in Case (ii). 
Case (iii). $i_{1}=n$. Now

$$
\begin{aligned}
x_{i_{1}} x_{i_{2}} \cdots x_{i_{n}} & =y_{m} a_{2 m} x_{i_{2}} \cdots x_{i_{n}} \\
& =y_{m} x_{1} x_{2} \cdots x_{n-1} a_{2 m} \quad(\text { since } U \text { satisfies (1)) } \\
& =y_{m} x_{1} x_{2} \cdots x_{n-1} a_{2 m-1} t_{m} \quad(\text { from equations }(2)) \\
& =y_{m} a_{2 m-1} x_{i_{2}} \cdots x_{i_{n}} t_{m} \quad(\text { since } U \text { satisfies }(1)) \\
& =y_{m-1} a_{2 m-2} x_{i_{2}} \cdots x_{i_{n}} t_{m} \quad(\text { from equations }(2)) \\
& =y_{m-1} x_{1} x_{2} \cdots x_{n-1} a_{2 m-2} t_{m} \quad(\text { since } U \text { satisfies (1)) } \\
& =y_{m-1} x_{1} x_{2} \cdots x_{n-1} a_{2 m-3} t_{m-1} \quad \text { (from equations (2)) } \\
& \vdots \\
& =y_{1} x_{1} x_{2} \cdots x_{n-1} a_{1} t_{1} \\
& =y_{1} a_{1} x_{i_{2}} \cdots x_{i_{n}} t_{1} \quad(\text { since } U \text { satisfies (1)) } \\
& =a_{0} x_{i_{2}} \cdots x_{i_{n}} t_{1} \quad(\text { from equations (2)) } \\
& =x_{1} x_{2} \cdots x_{n-1} a_{0} t_{1} \\
& =x_{1} x_{2} \cdots x_{n} \quad\left(\text { from equations (2), since } i_{1}=n\right),
\end{aligned}
$$

as required.

REMARK 1. A proof for Case (iii) could also be obtained from the proof for Case (ii) above by making the following conventions:

(a) the word $x_{j+1} \cdots x_{n}=1$;

(b) $b_{j+1}^{(k)}=\cdots=b_{n}^{(k)}=1$ and $t_{k}^{\prime}=t_{k}$ for $k=1,2, \ldots, m$;

(c) the vector

$$
\left(x_{1}, x_{2}, \ldots, x_{j-1}, a_{2 k-1}, b_{j+1}^{(k)}, \ldots, b_{n}^{(k)}\right)=\left(x_{1}, x_{2}, \ldots, x_{n-1}, a_{2 k-1}\right)
$$

for $k=1,2, \ldots, m$;

(d) the vector

$$
\left(x_{1}, x_{2}, \ldots, x_{j-1}, a_{2 k-2}, b_{j+1}^{(k)}, \ldots, b_{n}^{(k)}\right)=\left(x_{1}, x_{2}, \ldots, x_{n-1}, a_{2 k-2}\right)
$$

for $k=1,2, \ldots, m$.

So assume now that (1) is true for all $x_{i_{1}}, x_{i_{2}}, \ldots, x_{i_{q-1}} \in S$ and all $x_{i_{q}}, x_{i_{q+1}}, \ldots, x_{i_{n}}$ $\in U$. We prove from this assumption that (1) is true for all $x_{i_{1}}, x_{i_{2}}, \ldots, x_{i_{q}} \in S$ and for all $x_{i_{q+1}}, x_{i_{q+2}}, \ldots, x_{i_{n}} \in U$. We need not consider the case where $x_{i_{q}} \in U$, so we assume that $x_{i_{q}} \in S \backslash U$. As $x_{i_{q}} \in S \backslash U$ and $\operatorname{Dom}_{S}(U)=S$, by Result 1, we may let (2) be a zigzag of shortest possible length $m$ over $U$ with value $x_{i_{q}}$.

Put $j=i_{q}$ and $l=i_{q-1}$.

Case (i). $l=j-1$. Now

$$
\begin{aligned}
x_{i_{1}} x_{i_{2}} \cdots x_{i_{n}} & =x_{i_{1}} x_{i_{2}} \cdots x_{i_{q-1}}\left(y_{m} a_{2 m}\right) x_{i_{q+1}} \cdots x_{i_{n}} \\
& =x_{i_{1}} x_{i_{2}} \cdots\left(x_{i_{q-1}} y_{m}\right) a_{2 m} x_{i_{q+1}} \cdots x_{i_{n}} \\
& =x_{1} x_{2} \cdots x_{j-2}\left(x_{i_{q-1}} y_{m}\right) a_{2 m} x_{j+1} \cdots x_{n}
\end{aligned}
$$

(by the inductive hypothesis)

(Continues) 
as required.

$$
\begin{aligned}
& =x_{1} x_{2} \cdots x_{j-2} x_{i_{q-1}}\left(y_{m} a_{2 m}\right) x_{j+1} \cdots x_{n} \\
& =x_{1} x_{2} \cdots x_{j-2} x_{j-1} x_{j} x_{j+1} \cdots x_{n} \\
& \quad\left(\text { since } x_{i_{q}}=x_{j} \text { and } x_{i_{q-1}}=x_{j-1}\right),
\end{aligned}
$$

Case (ii). $l<j-1$ and $j<n$. Now

(6)

$$
\begin{aligned}
x_{i_{1}} & \cdots x_{i_{q-1}} x_{i_{q}} \cdots x_{i_{n}} \\
& =x_{i_{1}} \cdots x_{i_{q-1}} y_{m} a_{2 m} \cdots x_{i_{n}} \quad(\text { from equations }(2)) \\
& =w_{1}\left(x_{i_{1}}, x_{i_{2}}, \ldots, x_{i_{q-1}} y_{m}, a_{2 m}, \ldots, x_{i_{n}}\right) \\
& =w_{2}\left(x_{i_{1}}, x_{i_{2}}, \ldots, x_{i_{q-1}} y_{m}, a_{2 m}, \ldots, x_{i_{n}}\right) \quad(\text { by the inductive hypothesis }) \\
& =w_{2}\left(x_{i_{1}}, \ldots, x_{i_{q-1}} y_{m}, a_{2 m-1} t_{m}, \ldots, x_{i_{n}}\right) \quad(\text { from equations }(2)) \\
& =x_{1} x_{2} \cdots x_{l-1}\left(x_{i_{q-1}} y_{m}\right) x_{l+1} \cdots x_{j-1}\left(a_{2 m-1} t_{m}\right) z \quad\left(\text { where } z=x_{j+1} \cdots x_{n}\right) \\
& =x_{1} x_{2} \cdots x_{l-1}\left(x_{i_{q-1}} y_{m}\right) x_{l+1} \cdots x_{j-1} a_{2 m-1} b_{j+1}^{(m)} \cdots b_{n}^{(m)} t_{m}^{\prime} z \\
& \quad\left(\text { by Result } 4 \text { for some } b_{j+1}^{(m)}, \ldots, b_{n}^{(m)} \in U\right. \\
= & u_{2}\left(x_{1}, x_{2}, \ldots, x_{l-1}, x_{i_{q-1}} y_{m}, x_{l+1}, \ldots, x_{j-1}, a_{2 m-1}, b_{j+1}^{(m)}, \ldots, b_{n}^{(m)}\right) t_{m}^{\prime} z \\
& =u_{1}\left(x_{1}, \ldots, x_{l-1}, x_{i_{q-1}} y_{m}, x_{l+1}, \ldots, x_{j-1}, a_{2 m-1}, b_{j+1}^{(m)}, \ldots, b_{n}^{(m)}\right) t_{m}^{\prime} z
\end{aligned}
$$

(by the inductive hypothesis).

Since $u_{1}\left(z_{1}, z_{2}, \ldots, z_{n}\right)$ contains as a subword $z_{i_{q-1}} z_{i_{q}}$, the product (6) in $S$ contains $\left(x_{i_{q-1}} y_{m}\right) a_{2 m-1}$ which equals $\left(x_{i_{q-1}} y_{m-1}\right) a_{2 m-2}$ (from equations (2)). Thus the product (6) above equals

(7)

$$
\begin{aligned}
& u_{1}\left(x_{1}, \ldots, x_{l-1}, x_{i_{q-1}} y_{m-1}, x_{l+1}, \ldots, x_{j-1}, a_{2 m-2}, b_{j+1}^{(m)}, \ldots, b_{n}^{(m)}\right) t_{m}^{\prime} z \\
& \quad=u_{2}\left(x_{1}, \ldots, x_{l-1}, x_{i_{q-1}} y_{m-1}, x_{l+1}, \ldots, x_{j-1}, a_{2 m-2}, b_{j+1}^{(m)}, \ldots, b_{n}^{(m)}\right) t_{m}^{\prime} z
\end{aligned}
$$

(by the inductive hypothesis)

$$
\begin{aligned}
& =x_{1} x_{2} \cdots x_{l-1} x_{i_{q-1}} y_{m-1} x_{l+1} \cdots x_{j-1} a_{2 m-2} b_{j+1}^{(m)} \cdots b_{n}^{(m)} t_{m}^{\prime} z \\
& =x_{1} x_{2} \cdots x_{l-1} x_{i_{q-1}} y_{m-1} x_{l+1} \cdots x_{j-1} a_{2 m-2} t_{m} z \quad\left(\text { since } t_{m}=b_{j+1}^{(m)} \cdots b_{n}^{(m)} t_{m}^{\prime}\right) \\
& =x_{1} x_{2} \cdots x_{l-1} x_{i_{q-1}} y_{m-1} x_{l+1} \cdots x_{j-1} a_{2 m-3} t_{m-1} z \quad(\text { from equations }(2)) \\
& \vdots \\
& =x_{1} x_{2} \cdots x_{l-1} x_{i_{q-1}} y_{1} x_{l+1} \cdots x_{j-1} a_{1} t_{1} z \\
& =x_{1} x_{2} \cdots x_{l-1} x_{i_{q-1}} y_{1} x_{l+1} \cdots x_{j-1} a_{1} b_{j+1}^{(1)} \cdots b_{n}^{(1)} t_{1}^{\prime} z
\end{aligned}
$$

(by Result 4 for some $b_{j+1}^{(1)}, \ldots, b_{n}^{(1)} \in U, t_{1}^{\prime} \in S \backslash U$, since $t_{1} \in S \backslash U$ ) 


$$
\begin{aligned}
& =u_{2}\left(x_{1}, \ldots, x_{l-1}, x_{i_{q-1}} y_{1}, x_{l+1}, \ldots, x_{j-1}, a_{1}, b_{j+1}^{(1)}, \ldots, b_{n}^{(1)}\right) t_{1}^{\prime} z \\
& =u_{1}\left(x_{1}, \ldots, x_{l-1}, x_{i_{q-1}} y_{1}, x_{l+1}, \ldots, x_{j-1}, a_{1}, b_{j+1}^{(1)}, \ldots, b_{n}^{(1)}\right) t_{1}^{\prime} z
\end{aligned}
$$

(by the inductive hypothesis).

Now as $u_{1}\left(z_{1}, z_{2}, \ldots, z_{n}\right)$ contains $z_{i_{q-1}} z_{i_{q}}$ as a subword, the product (7) in $S$ contains $\left(x_{i_{q-1}} y_{1}\right) a_{1}$ which equals $x_{i_{q-1}} a_{0}$ (from equations (2)). Thus the product (7) above equals

$$
\begin{aligned}
& u_{1}\left(x_{1}, x_{2}, \ldots, x_{l-1}, x_{i_{q-1}}, x_{l+1}, \ldots, x_{j-1}, a_{0}, b_{j+1}^{(1)}, \ldots, b_{n}^{(1)}\right) t_{1}^{\prime} z \\
& =u_{2}\left(x_{1}, \ldots, x_{l-1}, x_{i_{q-1}}, x_{l+1}, \ldots, x_{j-1}, a_{0}, b_{j+1}^{(1)}, \ldots, b_{n}^{(1)}\right) t_{1}^{\prime} z \\
& \quad \text { (by the inductive hypothesis) } \\
& =x_{1} \cdots x_{l-1} x_{i_{q-1}} x_{l+1} \cdots x_{j-1} a_{0} b_{j+1}^{(1)} \cdots b_{n}^{(1)} t_{1}^{\prime} z \\
& =x_{1} \cdots x_{l-1} x_{i_{q-1}} x_{l+1} \cdots x_{j-1} a_{0} t_{1} x_{j+1} \cdots x_{n} \quad\left(\text { since } z=x_{j+1} \cdots x_{n}\right) \\
& =x_{1} x_{2} \cdots x_{n} \quad\left(\text { since } x_{i_{q-1}}=x_{l}, \text { and } a_{0} t_{1}=x_{i_{q}}=x_{j}\right)
\end{aligned}
$$

as required.

Case (iii). $l<j-1, j=n$. Now

$$
x_{i_{1}} x_{i_{2}} \cdots x_{i_{q-1}} x_{i_{q}} \cdots x_{i_{n}}
$$$$
=x_{i_{1}} x_{i_{2}} \cdots x_{i_{q-1}} y_{m} a_{2 m} \cdots x_{i_{n}} \text { (from equations (2)) }
$$$$
=x_{i_{1}} x_{i_{2}} \cdots\left(x_{i_{q-1}} y_{m}\right) a_{2 m} \cdots x_{i_{n}}
$$$$
=x_{1} x_{2} \cdots x_{l-1}\left(x_{i_{q-1}} y_{m}\right) x_{l+1} \cdots x_{n-1} a_{2 m} \text { (by the inductive hypothesis) }
$$

$=x_{1} x_{2} \cdots x_{l-1}\left(x_{i_{q-1}} y_{m}\right) x_{l+1} \cdots x_{n-1} a_{2 m-1} t_{m} \quad$ (from equations (2))

$=x_{i_{1}} x_{i_{2}} \cdots\left(x_{i_{q-1}} y_{m}\right) a_{2 m-1} \cdots x_{i_{n}} t_{m}$ (by the inductive hypothesis)

$=x_{i_{1}} x_{i_{2}} \cdots x_{i_{q-1}}\left(y_{m} a_{2 m-1}\right) \cdots x_{i_{n}} t_{m}$

$=x_{i_{1}} x_{i_{2}} \cdots x_{i_{q-1}}\left(y_{m-1} a_{2 m-2}\right) \cdots x_{i_{n}} t_{m} \quad$ (from equations (2))

$=x_{i_{1}} x_{i_{2}} \cdots\left(x_{i_{q-1}} y_{m-1}\right) a_{2 m-2} \cdots x_{i_{n}} t_{m}$

$=x_{1} x_{2} \cdots x_{l-1}\left(x_{i_{q-1}} y_{m-1}\right) x_{l+1} \cdots x_{n-1} a_{2 m-2} t_{m}$ (by the inductive hypothesis)

$=x_{1} x_{2} \cdots x_{l-1}\left(x_{i_{q-1}} y_{m-1}\right) x_{l+1} \cdots x_{n-1} a_{2 m-3} t_{m-1} \quad$ (from equations (2))

$\vdots$

$=x_{1} x_{2} \cdots x_{l-1}\left(x_{i_{q-1}} y_{1}\right) x_{l+1} \cdots x_{n-1} a_{1} t_{1}$

$=x_{i_{1}} x_{i_{2}} \cdots\left(x_{i_{q-1}} y_{1}\right) a_{1} \cdots x_{i_{n}} t_{1} \quad$ (by the inductive hypothesis)

$=x_{i_{1}} x_{i_{2}} \cdots x_{i_{q-1}}\left(y_{1} a_{1}\right) \cdots x_{i_{n}} t_{1}$

$=x_{i_{1}} x_{i_{2}} \cdots x_{i_{q-1}} a_{0} \cdots x_{i_{n}} t_{1}$

$=x_{1} x_{2} \cdots x_{l-1} x_{i_{q-1}} \cdots x_{n-1} a_{0} t_{1}$ (by the inductive hypothesis)

$=x_{1} x_{2} \cdots x_{n} \quad$ (from equations (2), since $i_{q}=n$ and $\left.i_{q-1}=l\right)$,

as required. 
REMARK 2. A proof for Case (iii) could also be obtained from the proof for Case (ii) by making the following conventions:

(a) the word $x_{j+1} \cdots x_{n}=1$,

(b) $b_{j+1}^{(k)}=\cdots=b_{n}^{(k)}=1$ and $t_{k}^{\prime}=t_{k}$ for $k=1,2, \ldots, m$;

(c) the vector

$$
\begin{aligned}
& \left(x_{1}, x_{2}, \ldots, x_{i_{q-1}} y_{k}, x_{l+1}, \ldots, x_{j-1}, a_{2 k-1}, b_{j+1}^{(k)}, \ldots, b_{n}^{(k)}\right) \\
& \quad=\left(x_{1}, x_{2}, \ldots, x_{i_{q-1}} y_{k}, \ldots, x_{n-1}, a_{2 k-1}\right) \text { for } k=1,2, \ldots, m ;
\end{aligned}
$$

and

$$
\begin{aligned}
\left(x_{1}, x_{2}, \ldots, x_{i_{q-1}} y_{k-1},\right. & \left.x_{l+1}, \ldots, x_{j-1}, a_{2 k-2}, b_{j+1}^{(k)}, \ldots, b_{n}^{(k)}\right) \\
= & \left(x_{1}, x_{2}, \ldots, x_{i_{q-1}} y_{k-1}, \ldots, x_{n-1}, a_{2 k-2}\right)
\end{aligned}
$$$$
\text { for } k=1,2, \ldots, m \text { and where } y_{0}=1 \text {. }
$$

Case (iv). $j+1<l<n$. We have

(8)

$$
\begin{aligned}
x_{i_{1}} x_{i_{2}} & \cdots x_{i_{n}}=x_{i_{1}} x_{i_{2}} \cdots x_{i_{q-1}} x_{i_{q}} \cdots x_{i_{n}} \\
& =x_{i_{1}} x_{i_{2}} \cdots x_{i_{q-1}} y_{m} a_{2 m} x_{i_{q+1}} \cdots x_{i_{n}} \quad(\text { from equations }(2)) \\
& =w_{1}\left(x_{i_{1}}, x_{i_{2}}, \ldots, x_{i_{q-1}} y_{m}, a_{2 m}, x_{i_{q+1}}, \ldots, x_{i_{n}}\right) \\
& =w_{2}\left(x_{i_{1}}, \ldots, x_{i_{q-1}} y_{m}, a_{2 m}, x_{i_{q+1}}, \ldots, x_{i_{n}}\right) \quad(\text { by the inductive hypothesis) } \\
& =x_{1} x_{2} \cdots x_{j-1} a_{2 m} x_{j+1} \cdots x_{l-1} x_{i_{q-1}} y_{m} x_{l+1} \cdots x_{n} \\
& =x_{1} x_{2} \cdots x_{j-1} a_{2 m-1} t_{m} x_{j+1} \cdots x_{l-1} x_{i_{q-1}} y_{m} x_{l+1} \cdots x_{n} \\
& =x_{1} x_{2} \cdots x_{j-1} a_{2 m-1} b_{j+1}^{(m)} \cdots b_{j+(l-j-1)}^{(m)} t_{m}^{\prime} x_{j+1} \cdots x_{l-1} x_{i_{q-1}} y_{m} x_{l+1} \cdots x_{n} \\
\left(\text { by Result } 4 \text { for some } b_{j+1}^{(m)}, \ldots, b_{j+(l-j-1)}^{(m)} \in U,\right. & \text { and } \left.t_{m} \in S \backslash U \text { since } t_{m} \in S \backslash U\right) \\
= & u_{2}\left(x_{1}, x_{2}, \ldots, x_{j-1}, a_{2 m-1}, b_{j+1}^{(m)}, \ldots, b_{j+(l-j-1)}^{(m)}, t_{m}^{\prime} x_{j+1} \cdots x_{i_{q-1}} y_{m},\right. \\
& \left.u_{l+1}, \ldots, x_{n}\right) \\
u_{1}\left(x_{1}, x_{2}, \ldots, x_{j-1}, a_{2 m-1}, b_{j+1}^{(m)}, \ldots, b_{j+(l-j-1)}^{(m)}, t_{m}^{\prime} x_{j+1} \cdots x_{i_{q-1}} y_{m},\right. & \left.x_{l+1}, \ldots, x_{n}\right)
\end{aligned}
$$

(by the inductive hypothesis).

Now since the word $u_{1}\left(z_{1}, z_{2}, \ldots, z_{n}\right)$ contains $z_{i_{q-1}} z_{i_{q}}$ as a subword, the product (8) in $S$ contains $\left(x_{i_{q-1}} y_{m}\right) a_{2 m-1}$ which equals $\left(x_{i_{q-1}} y_{m-1}\right) a_{2 m-2}$ (from equations (2)). Thus the product (8) above equals

$$
\begin{array}{r}
u_{1}\left(x_{1}, x_{2}, \ldots, x_{j-1}, a_{2 m-2}, b_{j+1}^{(m)}, \ldots, b_{j+(l-j-1)}^{(m)}, t_{m}^{\prime} x_{j+1}\right. \\
\left.\cdots x_{l-1} x_{i_{q-1}} y_{m-1}, x_{l+1}, \ldots, x_{n}\right)
\end{array}
$$




$$
\begin{aligned}
u_{2}\left(x_{1}, x_{2}, \ldots, x_{j-1}, a_{2 m-2}, b_{j+1}^{(m)}, \ldots, b_{j+(l-j-1)}^{(m)}, t_{m}^{\prime} x_{j+1}\right. \\
\left.\ldots x_{l-1} x_{i_{q-1}} y_{m-1}, x_{l+1}, \ldots, x_{n}\right)
\end{aligned}
$$

(by the inductive hypothesis)

$$
\begin{array}{r}
=x_{1} x_{2} \cdots x_{j-1} a_{2 m-2} b_{j+1}^{(m)} \cdots b_{j+(l-j-1)}^{(m)} t_{m}^{\prime} x_{j+1} \cdots x_{l-1} x_{i_{q-1}} y_{m-1} x_{l+1} \cdots x_{n} \\
=x_{1} x_{2} \cdots x_{j-1} a_{2 m-2} t_{m} x_{j+1} \cdots x_{l-1} x_{i_{q-1}} y_{m-1} x_{l+1} \cdots x_{n} \\
\left(\text { since } t_{m}=b_{j+1}^{(m)} \cdots b_{j+(l-j-1)}^{(m)} t_{m}^{\prime}\right) \\
=x_{1} x_{2} \cdots x_{j-1} a_{2 m-3} t_{m-1} x_{j+1} \cdots x_{l-1} x_{i_{q-1}} y_{m-1} x_{l+1} \cdots x_{n}
\end{array}
$$

(from equations (2))

$$
\begin{aligned}
& =x_{1} x_{2} \cdots x_{j-1} a_{1} t_{1} x_{j+1} \cdots x_{l-1} x_{i_{q-1}} y_{1} x_{l+1} \cdots x_{n} \\
& =x_{1} x_{2} \cdots x_{j-1} a_{1} b_{j+1}^{(1)} \cdots b_{j+(l-j-1)}^{(1)} t_{1}^{\prime} x_{j+1} \cdots x_{l-1} x_{i_{q-1}} y_{1} x_{l+1} \cdots x_{n}
\end{aligned}
$$

(by Result 4 for some $b_{j+1}^{(1)}, \ldots, b_{j+(l-j-1)}^{(1)} \in U$, and $t_{1}^{\prime} \in S \backslash U$, since $\left.t_{1} \in S \backslash U\right)$

$$
\begin{aligned}
& =u_{2}\left(x_{1}, \ldots, x_{j-1}, a_{1}, b_{j+1}^{(1)}, \ldots, b_{j+(l-j-1)}^{(1)}, t_{1}^{\prime} x_{j+1} \cdots x_{i_{q-1}} y_{1}, x_{l+1}, \ldots, x_{n}\right) \\
& =u_{1}\left(x_{1}, \ldots, x_{j-1}, a_{1}, b_{j+1}^{(1)}, \ldots, b_{j+(l-j-1)}^{(1)}, t_{1}^{\prime} x_{j+1} \cdots x_{i_{q-1}} y_{1}, x_{l+1}, \ldots, x_{n}\right) .
\end{aligned}
$$

As $u_{1}\left(z_{1}, z_{2}, \ldots, z_{n}\right)$ contains $z_{i_{q-1}} z_{i_{q}}$ as a subword, the product (9) in $S$ contains $\left(x_{i_{q-1}} y_{1}\right) a_{1}$ which equals $x_{i_{q-1}} a_{0}$ (from equations (2)). Thus the product (9) above equals

$$
\begin{aligned}
& u_{1}\left(x_{1}, \ldots, x_{j-1}, a_{0}, b_{j+1}^{(1)}, \ldots, b_{j+(l-j-1)}^{(1)}, t_{1}^{\prime} x_{j+1} \cdots x_{i_{q-1}}, x_{l+1}, \ldots, x_{n}\right) \\
& \quad=u_{2}\left(x_{1}, \ldots, x_{j-1}, a_{0}, b_{j+1}^{(1)}, \ldots, b_{j+(l-j-1)}^{(1)}, t_{1}^{\prime} x_{j+1} \cdots x_{i_{q-1}}, x_{l+1}, \ldots, x_{n}\right)
\end{aligned}
$$

(by the inductive hypothesis)

$$
\begin{aligned}
& =x_{1} x_{2} \cdots x_{j-1} a_{0} b_{j+1}^{(1)} \cdots b_{j+(l-j-1)}^{(1)} t_{1}^{\prime} x_{j+1} \cdots x_{l-1} x_{i_{q-1}} x_{l+1} \cdots x_{n} \\
& =x_{1} x_{2} \cdots x_{j-1} a_{0} t_{1} x_{j+1} \cdots x_{l-1} x_{i_{q-1}} x_{l+1} \cdots x_{n} \\
& \quad\left(\text { since } t_{1}=b_{j+1}^{(1)} \cdots b_{j+(l-j-1)}^{(1)} t_{1}^{\prime}\right) \\
& =x_{1} x_{2} \cdots x_{n}\left(\operatorname{since} x_{i_{q-1}}=x_{l}, \text { and } x_{i_{q}}=a_{0} t_{1} \doteq x_{j}\right),
\end{aligned}
$$

as required.

Case (v). $j+1=l$. Now $x_{i_{1}} x_{i_{2}} \cdots x_{i_{n}}$

$$
\begin{aligned}
& =x_{i_{1}} x_{i_{2}} \cdots x_{i_{q-1}} y_{m} a_{2 m} \cdots x_{i_{n}} \text { (from equations (2)) } \\
& =x_{i_{1}} x_{i_{2}} \cdots\left(x_{i_{q-1}} y_{m}\right) a_{2 m} \cdots x_{i_{n}} \\
& =x_{1} x_{2} \cdots x_{j-1} a_{2 m}\left(x_{i_{q-1}} y_{m}\right) x_{l+1} \cdots x_{n}
\end{aligned}
$$

(by the inductive hypothesis; if $l=n$, the product $x_{l+1} \cdots x_{n}=1$ ) 


$$
\begin{aligned}
& =x_{1} x_{2} \cdots x_{j-1} a_{2 m-1} t_{m}\left(x_{i_{q-1}} y_{m}\right) x_{l+1} \cdots x_{n} \quad \text { (from equations (2)) } \\
& =x_{1} x_{2} \cdots x_{j-1} a_{2 m-1}\left(t_{m} x_{i_{q-1}} y_{m}\right) x_{l+1} \cdots x_{n} \\
& =x_{i_{1}} x_{i_{2}} \cdots x_{i_{q-2}}\left(t_{m} x_{i_{q-1}} y_{m}\right) a_{2 m-1} x_{i_{q+1}} \cdots x_{i_{n}} \quad \text { (by the inductive hypothesis) } \\
& =x_{i_{1}} x_{i_{2}} \cdots x_{i_{q-2}}\left(t_{m} x_{i_{q-1}} y_{m-1}\right) a_{2 m-2} x_{i_{q+1}} \cdots x_{i_{n}} \quad \text { (from equations (2)) } \\
& =x_{1} x_{2} \cdots x_{j-1} a_{2 m-2}\left(t_{m} x_{i_{q-1}} y_{m-1}\right) x_{l+1} \cdots x_{n} \quad \text { (by the inductive hypothesis) } \\
& =x_{1} x_{2} \cdots x_{j-1} a_{2 m-3}\left(t_{m-1} x_{i_{q-1}} y_{m-1}\right) x_{l+1} \cdots x_{n} \quad \text { (from equations (2)) } \\
& \vdots \\
& =x_{1} x_{2} \cdots x_{j-1} a_{1}\left(t_{1} x_{i_{q-1}} y_{1}\right) x_{l+1} \cdots x_{n} \\
& =x_{i_{1}} x_{i_{2}} \cdots x_{i_{q-2}}\left(t_{1} x_{i_{q-1}} y_{1}\right) a_{1} x_{i_{q+1}} \cdots x_{i_{n}} \quad \text { (by the inductive hypothesis) } \\
& =x_{i_{1}} x_{i_{2}} \cdots x_{i_{q-2}}\left(t_{1} x_{i_{q-1}}\right) a_{0} x_{i_{q+1}} \cdots x_{i_{n}} \quad \text { (from equations (2)) } \\
& =x_{1} x_{2} \cdots x_{j-1} a_{0}\left(t_{1} x_{i_{q-1}}\right) x_{l+1} \cdots x_{n} \quad \text { (by the inductive hypothesis) } \\
& =x_{1} x_{2} \cdots x_{n}\left(\text { from equations }(2) \text { and } i_{q-1}=l=j+1\right),
\end{aligned}
$$

as required.

Finally, a proof in the remaining Case (vi), namely when $j+1<l$ and $l=n$, can be obtained from the proof for Case (iv) above by making the following conventions:

(a) the word $x_{l+1} \cdots x_{n}=1$;

(b) the vector

$$
\begin{aligned}
& \left(x_{1}, x_{2}, \ldots, x_{j-1}, a_{2 k-1}, b_{j+1}^{(k)}, \ldots, b_{j+(l-j-1)}^{(k)}, t_{k}^{\prime} x_{j+1} \cdots x_{l-1} x_{i_{q-1}} y_{k}, x_{l+1}, \ldots, x_{n}\right) \\
& \quad=\left(x_{1}, x_{2}, \ldots, x_{j-1}, a_{2 k-1}, b_{j+1}^{(k)}, \ldots, b_{n-1}^{(k)}, t_{k}^{\prime} x_{j+1} \cdots x_{n-1} x_{i_{q-1}} y_{k}\right)
\end{aligned}
$$

for $k=1,2, \ldots, m$;

(c) the vector

$$
\begin{gathered}
\left(x_{1}, x_{2}, \ldots, x_{j-1}, a_{2 k-2}, b_{j+1}^{(k)}, \ldots, b_{j+(l-j-1)}^{(k)}, t_{k}^{\prime} x_{j+1} \cdots x_{l-1} x_{i_{q-1}} y_{k-1}, x_{l+1}, \ldots, x_{n}\right) \\
=\left(x_{1}, x_{2}, \ldots, x_{j-1}, a_{2 k-2}, b_{j+1}^{(k)}, \ldots, b_{n-1}^{(k)}, t_{k}^{\prime} x_{j+1} \cdots x_{n-1} x_{i_{q-1}} y_{k-1}\right)
\end{gathered}
$$

for $k=1,2, \ldots, m$ and where $y_{0}=1$.

This completes the proof of Theorem 3.1.

The following corollary gives a sufficient condition for $\operatorname{Dom}_{S}(U)$ to satisfy any permutation identity that $U$ satisfies and, thus, generalizes [9, Corollary 2.5] from commutativity to any permutation identity.

Corollary 3.2 (TO THE PRoOf of TheOrem 3.1). Let $U$ and $S$ be any semigroups with $U$ a subsemigroup of $S$. Let $U$ satisfy a permutation identity (1). If for all $s \in S \backslash U, s=a s^{\prime}$ for some $a \in U$ and $s^{\prime} \in S$, then $\operatorname{Dom}_{S}(U)$ also satisfies the permutation identity (1) satisfied by $U$. 
REMARK 3. Theorem 3.1 generalizes [9, Corollary 2.5], which stated that commutativity is preserved under epis of semigroups.

EXample (P. M. Higgins, verbal COMmunication). This shows that the nontrivial permutation identities other than commutativity are not carried over to dominions.

Let $F_{X}$ be the free semigroup on a countable infinite set $X=\left\{x_{1}, x_{2}, \ldots\right\}$. Let $T=\langle Y\rangle$, the subsemigroup of $F_{X}$ generated by the set $Y$, where

$$
Y=\bigcup_{n=0}^{\infty}\left\{x_{3 n+1} x_{3 n+2}, x_{3 n+2}, x_{3 n+2} x_{3 n+3}\right\} \text {. }
$$

Put $S=F_{X / \rho}$ and $\bar{T}=T^{\rho^{\natural}}$, where $\rho$ is the congruence generated by the relation $\rho_{0}$ which consists of the pairs $\left(u_{1} u_{2} \cdots u_{n}, u_{i_{1}} u_{i_{2}} \cdots u_{i_{n}}\right)$ with $u_{j} \in T$ for $j=1,2, \ldots, n$, and where $i$ is a fixed nontrivial permutation of the set $\{1,2, \ldots, n\}$ with $n \geqslant 3$. It is easy to see that for each $n=0,1,2, \ldots,\left(x_{3 n+1} x_{3 n+2} x_{3 n+3}\right) \rho \in \operatorname{Dom}_{S}(\bar{T})$. Now we show that $\operatorname{Dom}_{S}(\bar{T})$ does not satisfy the permutation identity corresponding to the permutation $i$.

To see this consider the product $\left(x_{1} x_{2} x_{3}\right)\left(x_{4} x_{5} x_{6}\right) \cdots\left(x_{3 n+1} x_{3 n+2} x_{3 n+3}\right)$ in $F_{X}$. Since no $n$ members of $T$ occur consecutively in this word, no elementary $\rho_{0}$ transition is possible from this base and hence $\operatorname{Dom}_{S}(\bar{T})$ does not satisfy the permutation identity corresponding to the permutation $i$.

4. Epimorphically closed permutative varieties. In Theorems 4.1 and 4.4 the bracketed statements are dual to the other statements.

THEOREM 4.1. Let equation (1) be any permutation identity with $n \geqslant 3$ and such that $i_{n} \neq n\left[i_{1} \neq 1\right]$. Then all identities, in conjunction with (1), are preserved under epis.

Proof. Take any identity

$$
u\left(x_{1}, x_{2}, \ldots, x_{p}\right)=v\left(x_{1}, x_{2}, \ldots, x_{p}\right)
$$

and any semigroups $U$ and $S$ such that $U$ is a subsemigroup of $S, U$ satisfies (1) and (10), and $\operatorname{Dom}_{S}(U)=S$.

By Theorem 3.1, $S$ satisfies (1). Now we show that $S$ satisfies (10). Since $S$ satisfies (1), by the dual of Result 3, $S$ also satisfies the permutation identity

$$
x_{1} x_{2}, \ldots, x_{n} x y=x_{1} x_{2} \cdots x_{n} y x .
$$

LEMMA 4.2. Take any word $w$ in variables $x_{1}, x_{2}, \ldots, x_{k}$ say, any $a_{1}, a_{2}, \ldots, a_{k} \in U$, and any $t_{1}, t_{2}, \ldots, t_{k} \in S^{1}$ such that if $t_{i} \in S$, then $a_{i}=y_{i} b_{i}$ for some $y_{i} \in S \backslash U$, $b_{i} \in S(i=1,2, \ldots, k)$. Then

$$
w\left(a_{1} t_{1}, a_{2} t_{2}, \ldots, a_{k} t_{k}\right)=w\left(a_{1}, a_{2}, \ldots, a_{k}\right) w\left(t_{1}, t_{2}, \ldots, t_{k}\right) .
$$

Proof. Let $x_{q}$ be the first variable appearing in $w$ for which $t_{q} \in S$ (whence $a_{q}=y_{q} b_{q}$ for some $y_{q} \in S \backslash U, b_{q} \in S$ ). Then

$$
\begin{aligned}
w\left(a_{1} t_{1}, a_{2} t_{2}, \ldots, a_{k} t_{k}\right) & =w\left(a_{1} t_{1}, a_{2} t_{2}, \ldots, y_{q} b_{q} t_{q}, \ldots, a_{k} t_{k}\right) \\
& =w\left(a_{1}, a_{2}, \ldots, y_{q} b_{q}, \ldots, a_{k}\right) w\left(t_{1}, t_{2}, \ldots, t_{k}\right) \quad \text { (by Result 5) } \\
& =w\left(a_{1}, a_{2}, \ldots, a_{k}\right) w\left(t_{1}, t_{2}, \ldots, t_{k}\right),
\end{aligned}
$$

as required. 
We return to the proof of Theorem 4.1. Take any $d_{1}, d_{2}, \ldots, d_{p} \in S$. If some $d_{i} \in U$, there is a zigzag in $S^{1}$ over $u$ with value $d_{i}$, namely

$$
d_{i}=d_{i} 1=1 d_{i} 1=1 d_{i} \text {. }
$$

Now $d_{1}, d_{2}, \ldots, d_{p}$ all have zigzags over $U$ in $S^{1}$ of some common length [10, Lemma 4.2], say

$$
\begin{aligned}
& d_{i}=a_{0}^{(i)} t_{1}^{(i)}, \quad a_{0}^{(i)}=y_{1}^{(i)} a_{1}^{(i)}, \\
& y_{k}^{(i)} a_{2 k}^{(i)}=y_{k+1}^{(i)} a_{2 k+1}^{(i)}, \quad a_{2 k-1}^{(i)} t_{k}^{(i)}=a_{2 k}^{(i)} t_{k+1}^{(i)} \\
& \quad(i=1,2, \ldots, p, k=1,2, \ldots, m-1), \\
& a_{2 m-1}^{(i)} t_{m}^{(i)}=a_{2 m}^{(i)}, \quad y_{m}^{(i)} a_{2 m}^{(i)}=d_{i},
\end{aligned}
$$

where $a_{j}^{(i)} \in U(i=1,2, \ldots, p, j=0,1,2, \ldots, 2 m)$ and $t_{q}^{(i)}, y_{q}^{(i)} \in S^{1} \quad(i=$ $1,2, \ldots, p, q=1,2, \ldots, m)$, and further, for each $d_{i} \in S \backslash U$ we can assume that $t_{q}^{(i)}$, $y_{q}^{(i)} \in S \backslash U$ (from the proof of [10, Lemma 4.2]).

In the following, we shall make free use of Lemma 4.2 without explicit mention. We put $\tilde{x}=\left(x_{1}, x_{2}, \ldots, x_{p}\right)$. In this notation, the identity $(10)$ is simply $u(\tilde{x})=v(\tilde{x})$.

Put

$$
\begin{array}{ll}
\tilde{d}=\left(d_{1}, d_{2}, \ldots, d_{p}\right), & \\
\tilde{a}_{k}=\left(a_{k}^{(1)}, a_{k}^{(2)}, \ldots, a_{k}^{(p)}\right) & (k=0,1,2, \ldots, 2 m), \\
\tilde{t}_{q}=\left(t_{q}^{(1)}, t_{q}^{(2)}, \ldots, t_{q}^{(p)}\right) & (q=1,2, \ldots, m), \\
\tilde{y}_{q}=\left(y_{q}^{(1)}, y_{q}^{(2)}, \ldots, y_{q}^{(p)}\right) & (q=1,2, \ldots, m) .
\end{array}
$$

We wish to show that $u(\tilde{d})=v(\tilde{d})$.

By [10, Lemma 4.3], $\tilde{d} \in S^{p}$ is in the dominion of $U^{p}$ in $\left(S^{1}\right)^{p}$, where $T^{\gamma}$, for any semigroup $T$ and any integer $\gamma \geqslant 2$, denotes the cartesian product of $\gamma$-copies of the semigroup $T ; \tilde{d}$ has the following zigzag of length $m$ :

$$
\begin{aligned}
& \tilde{d}=\tilde{a}_{0} \tilde{t}_{1}, \quad \tilde{a}_{0}=\tilde{y}_{1} \tilde{a}_{1}, \\
& \tilde{y}_{k} \tilde{a}_{2 k}=\tilde{y}_{k+1} \tilde{a}_{2 k+1}, \quad \tilde{a}_{2 k-1} \tilde{t}_{k}=\tilde{a}_{2 k} \tilde{t}_{k+1} \quad(k=1,2, \ldots, m-1), \\
& \tilde{a}_{2 m-1} \tilde{t}_{m}=\tilde{a}_{2 m}, \quad \tilde{y}_{m} \tilde{a}_{2 m}=\tilde{d},
\end{aligned}
$$

where $\tilde{a}_{t} \in U^{p}(t \in 0,1,2, \ldots, 2 m)$, and $\tilde{y}_{q}, \tilde{t}_{q} \in\left(S^{1}\right)^{p}(q=1,2, \ldots, m)$.

LEMMA 4.3. Let the word $v$ in (10) begin with $x_{j}$, say. If $d_{j} \in S \backslash U$, then $u(\tilde{d})=v(\tilde{d})$.

ProOF.

$$
\begin{aligned}
u(\tilde{d}) & =u\left(\tilde{a}_{0} \tilde{t}_{1}\right) \quad(\text { from equations }(14)) \\
& =u\left(\tilde{a}_{0}\right) u\left(\tilde{t}_{1}\right) \quad\left(\text { by Lemma } 4.2, \text { since each } a_{0}^{(i)}=y_{1}^{(i)} a_{1}^{(i)}\right) \\
& =v\left(\tilde{y}_{1} \tilde{a}_{1}\right) u\left(\tilde{t}_{1}\right) \quad(\text { since } U \text { satisfies }(10)) \\
& =v\left(\tilde{y}_{1}\right) v\left(\tilde{a}_{1}\right) u\left(\tilde{t}_{1}\right) \quad\left(\text { by Result } 5, \text { since } y_{1}^{(j)} \in S \backslash U\right) \\
& =v\left(\tilde{y}_{1}\right) u\left(\tilde{a}_{1}\right) u\left(\tilde{t}_{1}\right) \quad(\text { since } U \text { satisfies }(10))
\end{aligned}
$$




$$
\begin{aligned}
& =v\left(\tilde{y}_{1}\right) u\left(\tilde{a}_{1} \tilde{t}_{1}\right) \quad\left(\text { by Result } 5, \text { since } y_{1}^{(j)} \in S \backslash U\right) \\
& =v\left(\tilde{y}_{1}\right) u\left(\tilde{a}_{2} \tilde{t}_{2}\right) \quad(\text { from equation }(14)) \\
& \vdots \\
& =v\left(\tilde{y}_{m-1}\right) u\left(\tilde{a}_{2 m-2} \tilde{t}_{m}\right) \\
& =v\left(\tilde{y}_{m-1}\right) u\left(\tilde{a}_{2 m-2}\right) u\left(\tilde{t}_{m}\right) \quad\left(\text { by Result } 5, \text { since } y_{m-1}^{(j)} \in S \backslash U\right) \\
& =v\left(\tilde{y}_{m-1}\right) v\left(\tilde{a}_{2 m-2}\right) u\left(\tilde{t}_{m}\right) \quad(\text { since } U \text { satisfies }(10)) \\
& =v\left(\tilde{y}_{m-1} \tilde{a}_{2 m-2}\right) u\left(\tilde{t}_{m}\right) \quad\left(\text { by Result } 5, \text { since } y_{m-1}^{(j)} \in S \backslash U\right) \\
& =v\left(\tilde{y}_{m} \tilde{a}_{2 m-1}\right) u\left(\tilde{t}_{m}\right) \quad(\text { from equations }(14)) \\
& \left.=v\left(\tilde{y}_{m}\right) v\left(\tilde{a}_{2 m-1}\right) u\left(\tilde{t}_{m}\right) \quad \text { (by Result } 5, \text { since } y_{m}^{(j)} \in S \backslash U\right) \\
& =v\left(\tilde{y}_{m}\right) u\left(\tilde{a}_{2 m-1} \tilde{t}_{m}\right) \quad\left(\text { by Result } 5, \text { since } y_{m}^{(j)} \in S \backslash U,\right. \\
& =v\left(\tilde{y}_{m}\right) u\left(\tilde{a}_{2 m}\right) \quad(\text { from equations }(14)) \\
& =v\left(\tilde{y}_{m} \tilde{a}_{2 m}\right) \quad\left(\text { by Result } 5, \text { since } y_{m}^{(j)} \in S \backslash U \text { and } U \text { satisfies }(10)\right) \\
& =v(\tilde{d}) \quad(\text { from equations }(14)) .
\end{aligned}
$$

This completes the proof of Lemma 4.3.

We return again to the proof of Theorem 4.1. We regard the variables $x_{1}$, $x_{2}, \ldots, x_{n}$ as being "replaced by" $d_{1}, d_{2}, \ldots, d_{n}$ respectively, and it will be convenient for us to use the phrase "replaced by" in our proof. If all the variables in $u$ and $v$ are replaced from $U$, then $u(\tilde{d})=v(\tilde{d})$ as required; hence we assume that in $v$, say, not every variable is replaced from $U$.

By Lemma 4.3, if the first variable of $v$ is replaced by an element of $S \backslash U$, then we have the required result again. Hence we consider now the case where further the first variable of $v$ is replaced by an element of $U$. Then

$$
v(\tilde{x})=v_{1}(\tilde{x}) v_{2}(\tilde{x})
$$

for some words $v_{1}$ and $v_{2}$ in the variables $x_{1}, x_{2}, \ldots, x_{p}$, where $v_{1}$ is of the maximum length such that all the variables of $v_{1}$ are replaced by elements of $U$ (the word $v_{1}$ is nonempty and not all the variables $x_{1}, x_{2}, \ldots, x_{p}$ appear in $v_{1}$ ). Let the first variable of $v_{2}(\tilde{x})$ be $x_{l}$, say (that is, $x_{l}$ is the first variable appearing in $v(\tilde{x})$ which is replaced by an element of $S \backslash U$ ).

For any $i$, if $d_{i} \in S \backslash U$ then $y_{j}^{(i)} \in S \backslash U$ for $j=1,2, \ldots, m$. Therefore, by Results 1 and 2, for $d_{i} \in S \backslash U$, we can write

$$
y_{j}^{(i)}=b_{j}^{(i)} \bar{y}_{j}^{(i)} \text { and } b_{j}^{(i)}=z_{j}^{(i)} c_{j}^{(i)} \text { for } j=1,2, \ldots, m,
$$

for some $b_{j}^{(i)}, c_{j}^{(i)} \in U, \bar{y}_{j}^{(i)}, z_{j}^{(i)} \in S \backslash U$. For each $d_{i} \in U$, we put

$$
b_{j}^{(i)}=c_{j}^{(i)}=\bar{y}_{j}^{(i)}=z_{j}^{(i)}=1 .
$$


In addition to the notations (13), we shall also use the following:

$$
\begin{array}{lc}
\tilde{b}_{q}=\left(b_{q}^{(1)}, b_{q}^{(2)}, \ldots, b_{q}^{(p)}\right) & (q=1,2, \ldots, m), \\
\bar{y}_{q}=\left(\bar{y}_{q}^{(1)}, \bar{y}_{q}^{(2)}, \ldots, \bar{y}_{q}^{(p)}\right) & (q=1,2, \ldots, m), \\
\tilde{c}_{q}=\left(c_{q}^{(1)}, c_{q}^{(2)}, \ldots, c_{q}^{(p)}\right) & (q=1,2, \ldots, m), \\
\tilde{z}_{q}=\left(z_{q}^{(1)}, z_{q}^{(2)}, \ldots, z_{q}^{(p)}\right) & (q=1,2, \ldots, m) .
\end{array}
$$

Now from equations (17) and (18) we have

$$
\tilde{y}_{q}=\tilde{b}_{q} \bar{y}_{q}=\tilde{z}_{q} \tilde{c}_{q} \bar{y}_{q} .
$$

Now

$$
\begin{aligned}
u(\tilde{d}) & =u\left(\tilde{a}_{0} \tilde{t}_{1}\right) \quad(\text { from equations }(14)) \\
& =u\left(\tilde{a}_{0}\right) u\left(\tilde{t}_{1}\right) \quad\left(\text { by Lemma } 4.2 \text { since } a_{0}^{(i)}=y_{1}^{(i)} a_{1}^{(i)} \text { for } i=1,2, \ldots, p\right) \\
& =v\left(\tilde{a}_{0}\right) u\left(\tilde{t}_{1}\right) \quad(\text { since } U \text { satisfies }(10)) \\
& =v\left(\tilde{y}_{1} \tilde{a}_{1}\right) u\left(\tilde{t}_{1}\right) \quad(\text { from equations }(14)) \\
& \vdots \\
= & v\left(\tilde{y}_{i} \tilde{a}_{2 i-1}\right) u\left(\tilde{t}_{i}\right) \quad(\text { this equality is essentially an inductive assumption; } \\
& \left.\quad \text { we now obtain equality with } v\left(\tilde{y}_{i+1} \tilde{a}_{2 i+1}\right) u\left(\tilde{t}_{i+1}\right)\right) \\
= & v_{1}\left(\tilde{y}_{i} \tilde{a}_{2 i-1}\right) v_{2}\left(\tilde{y}_{1} \tilde{a}_{2 i-1}\right) u\left(\tilde{t}_{1}\right) \quad(\text { from equation }(15))
\end{aligned}
$$

(since all variables of $v_{1}$ are replaced from $U$ )

$=v\left(\tilde{b}_{i} \tilde{a}_{2 i-1}\right) u\left(\tilde{t}_{i}\right) v_{2}\left(\bar{y}_{i}\right) \quad($ from equation $(15))$

$=u\left(\tilde{b}_{i} \tilde{a}_{2 i-1}\right) u\left(\tilde{t}_{i}\right) v_{2}\left(\bar{y}_{i}\right) \quad($ since $U$ satisfies (10))

$=u\left(\tilde{b}_{i} \tilde{a}_{2 i-1} \tilde{t}_{i}\right) v_{2}\left(\bar{y}_{i}\right) \quad$ (by Lemma 4.2 , since if any $t_{i}^{(j)} \in S \backslash U$ for any $j$, then $b_{i}^{(j)}=z_{i}^{(j)} c_{i}^{(j)}$ with $z_{i}^{(j)} \in S \backslash U$ from equation (16))

$=u\left(\tilde{b}_{i} \tilde{a}_{2 i} \tilde{t}_{i+1}\right) v_{2}\left(\bar{y}_{i}\right) \quad$ (from equations (14)) 


$$
\begin{aligned}
&= u\left(\tilde{b}_{i} \tilde{a}_{2 i}\right) u\left(\tilde{t}_{i+1}\right) v_{2}\left(\bar{y}_{i}\right) \quad\left(\text { by Lemma } 4.2, \text { since if any } t_{i+1}^{(j)} \in S \backslash U \text { for any } j,\right. \\
& \text { then } b_{i}^{(j)}=z_{i}^{(j)} c_{i}^{(j)} \text { with } z_{i}^{(j)} \in S \backslash U \text { from equation (16)) } \\
&=v\left(\tilde{b}_{i} \tilde{a}_{2 i}\right) u\left(\tilde{t}_{i+1}\right) v_{2}\left(\bar{y}_{i}\right) \quad(\text { since } U \text { satisfies }(10)) \\
&=v_{1}\left(\tilde{b}_{i} \tilde{a}_{2 i}\right) v_{2}\left(\tilde{b}_{i} \tilde{a}_{2 i}\right) u\left(\tilde{t}_{i+1}\right) v_{2}\left(\bar{y}_{i}\right) \quad(\text { from equation }(15)) \\
&=v_{1}\left(\tilde{a}_{2 i}\right) v_{2}\left(\tilde{b}_{i} \tilde{a}_{2 i}\right) u\left(\tilde{t}_{i+1}\right) v_{2}\left(\bar{y}_{i}\right)
\end{aligned}
$$

(since all variables of $v_{1}$ are replaced from $U$ )

$=v_{1}\left(\tilde{a}_{2 i}\right) v_{2}\left(\tilde{b}_{i}\right) v_{2}\left(\bar{y}_{i}\right) v_{2}\left(\tilde{a}_{2 i}\right) u\left(\tilde{t}_{i+1}\right) \quad$ (by Result 5, since $b_{i}^{(l)}=z_{i}^{(l)} c_{i}^{(l)}$ and $z_{i}^{(l)} \in S \backslash U$ from equation (16))

$=v_{1}\left(\tilde{a}_{2 i}\right) v_{2}\left(\tilde{b}_{i} \bar{y}_{i} \tilde{a}_{2 i}\right) u\left(\tilde{t}_{i+1}\right) \quad$ (by Result 5, since $b_{i}^{(l)}=z_{i}^{(l)} c_{i}^{(l)}$ and $z_{i}^{(l)} \in S \backslash U$ from equation (16))

$=v_{1}\left(\tilde{a}_{2 i}\right) v_{2}\left(\tilde{y}_{i} \tilde{a}_{2 i}\right) u\left(\tilde{t}_{i+1}\right) \quad($ from equation (19))

$=v_{1}\left(\tilde{y}_{i} \tilde{a}_{2 i}\right) v_{2}\left(\tilde{y}_{i} \tilde{a}_{2 i}\right) u\left(\tilde{t}_{i+1}\right) \quad$ (since all variables of $v_{1}$ are replaced from $U$ )

$=v\left(\tilde{y}_{i} \tilde{a}_{2 i}\right) u\left(\tilde{t}_{i+1}\right)$

$=v\left(\tilde{y}_{i+1} \tilde{a}_{2 i+1}\right) u\left(\tilde{t}_{i+1}\right) \quad($ if $i \leqslant m-1)$

$\vdots$

$=v\left(\tilde{y}_{m} \tilde{a}_{2 m}\right)=v(\tilde{d})$,

as required. This completes the proof of Theorem 4.1.

A restatement of Theorem 4.1 in terms of permutative varieties gives us a generalization of the author's result [10, Theorem 4.1] which states that all commutative varieties are closed under epis.

THEOREM 4.4. Let $\mathscr{V}$ be the permutative variety defined by a permutation identity (1) such that $i_{n} \neq n\left[i_{1} \neq 1\right]$. Then all subvarieties of the variety $\mathscr{V}$ are closed under epis.

Call an identity $u=v$ epimorphically stable or stable under epis if all identities in conjunction with it are preserved under epis, by which we mean that if $U$ is any semigroup satisfying $u=v$ and $S$ is any epimorphic extension of $U$, then $S$ satisfies all the identities satisfied by $U$.

In his paper [6], P. M. Higgins has provided an example showing that some permuation identities are not epimorphically stable, namely those permutation identities which are consequences of the normality identity $x y z w=x z y w$. Theorem 4.4 gives a sufficient condition for permutation identities to be epimorphically stable. So as a joint result, in the following theorem, we determine all the permutation identities which are epimorphically stable.

THEOREM 4.5. A permutation identity (1) is epimorphically stable if and only if $i_{n} \neq n$ or $i_{1} \neq 1$.

Proposition 4.6. Let $U$ and $S$ be any semigroups with $U$ a subsemigroup of $S$ and such that $\operatorname{Dom}_{S}(U)=S$. Take any $d \in S \backslash U$. Let (2) be a zigzag of length $m$ over $U$ with value $d$ with $y_{1} \in S \backslash U$ (for example if the zigzag is of shortest possible length). If $U$ satisfies any nontrivial permutation identity, then $d^{k}=a_{0}^{k} t_{1}^{k}$ for any positive integer $k$. 
Proof. We have

$$
\begin{aligned}
d^{k} & =\left(a_{0} t_{1}\right)^{k}=a_{0} t_{1}\left(a_{0} t_{1}\right)^{k-2} a_{0} t_{1} \quad\left(\text { if } k-2=0,\left(a_{0} t_{1}\right)^{k-2}=1\right) \\
& =y_{1} a_{1} t_{1}\left(a_{0} t_{1}\right)^{k-2} a_{0} t_{1} \quad(\text { from equations }(2)) \\
& =y_{1} a_{1} a_{0}^{k-1} t_{1}^{k} \quad\left(\text { by Result } 5, \text { since } y_{1}, t_{1} \in S \backslash U\right) \\
& =a_{0}^{k} t_{1}^{k},
\end{aligned}
$$

as required.

In general, for any nontrivial permutation identity $I$, say, we have not yet been able to determine completely which identities are preserved under epis in conjunction with $I$. However, we have the following

THEOREM 4.7. Let equation (1) be any nontrivial permutation identity. Then a nontrivial semigroup identity I (one which is not satisfied by the class of all semigroups) is preserved under epis in conjunction with (1) if I has one of the following forms:

(i) at least one side of I has no repeated variable;

(ii) $x^{p}=y^{q}, p, q>0$

(iii) $x_{1}^{p} x_{2}^{p} \cdots x_{l}^{p}=x_{1}^{q} x_{2}^{q} \cdots x_{l}^{q}, p, q>0, l \geqslant 1$;

(iv) $x^{p} y^{q}=y^{r} x^{s}, p, q, r, s>0$;

(v) $x^{p}=0, p>0$;

(vi) $x^{p} y^{q}=0, p, q>0$.

REMARK 4 . We regard $u=0$ (for some nonempty word $u$ ) as a semigroup identity: We define it to mean the conjunction of the two identities $u y=u=y u$ (in each case $y$ is a variable not occurring in the word $u$ ).

Proof. Take any semigroups $U$ and $S$ with $U$ epimorphically embedded in $S$, and such that $U$ (and hence, $S$, by Theorem 3.1) satisfies the identity (1). We show that each of the identities (i) to (vi) satisfied by $U$ is also satisfied by $S$.

(i) That $S$ satisfies (i), if $U$ does, follows from [11, Theorem 3.5].

(ii) Assume $U$ satisfies (ii). Then for all $u, v \in U$ we have $u^{p}=v^{q}=v^{p}=u^{q}$.

Take any $x, y \in S$. We assume first that $x \in S \backslash U$. By Result 1, we may let (2) be a zigzag of shortest possible length $m$ over $U$ with value $x$. Then

$$
\begin{aligned}
x^{p} & =a_{0}^{p} t_{1}^{p} \quad(\text { by Proposition } 4.6 \text { and equations }(2)) \\
& =\left(y_{1} a_{1}^{2}\right)^{p} t_{1}^{p} \quad\left(\text { since } y_{1} a_{1}^{2}=y_{1} a_{1} a_{1}=a_{0} a_{1} \in U\right) \\
& =y_{1}^{p} a_{1}^{p}\left(a_{1} t_{1}\right)^{p} \quad\left(\text { by Result } 6, \text { since } y_{1}, t_{1} \in S \backslash U\right) \\
& =y_{1}^{p} a_{1}^{p}\left(a_{2} t_{2}\right)^{p} \quad(\text { from equations }(2)) \\
& =y_{1}^{p} a_{1}^{p} a_{2}^{p} t_{2}^{p} \quad\left(\text { by Result } 6, \text { since } y_{1}, t_{2} \in S \backslash U\right) \\
& =y_{1}^{p} a_{1}^{p} a_{3}^{p} t_{2}^{p} \quad\left(\text { since } a_{2}^{p}=a_{3}^{p}\right) \\
& \vdots \\
& =y_{1}^{p} a_{1}^{p} a_{2 m-1}^{p} t_{m}^{p} \\
& =\left(y_{1} a_{1} a_{2 m-1} t_{m}\right)^{p} \quad\left(\text { by Result } 6, \text { since } y_{1}, t_{m} \in S \backslash U\right) \\
& =\left(y_{1} a_{1} a_{2 m}\right)^{p} \quad(\text { from equations }(2)) \\
& =\left(a_{0} a_{2 m}\right)^{p}=u^{p} \quad \text { for all } u \in U .
\end{aligned}
$$


Hence $x^{p}=u^{p}$ for all $x \in S, u \in U$ and likewise $y^{q}=u^{q}$ for all $y \in S$ and $u \in U$. Therefore $x^{p}=u^{p}=u^{q}=y^{q}$, as required.

(iii) Assume $U$ satisfies (iii). For $k=1,2, \ldots, l$, consider the word $x_{1}^{p} x_{2}^{p} \cdots x_{k}^{p}$ of length $k p$. We shall prove that $S$ satisfies (iii) by induction on the length of these words, assuming that the remaining elements $x_{k+1}, \ldots, x_{l} \in U$.

First for $k=0$, the equation (iii) is satisfied vacuously. So assume next that (iii) is true for all $x_{1}, x_{2}, \ldots, x_{k-1} \in S$ and all $x_{k}, x_{k+1}, \ldots, x_{l} \in U$. We prove from this assumption that (iii) is true for all $x_{1}, x_{2}, \ldots, x_{k} \in S$ and for all $x_{k+1}, x_{k+2}, \ldots, x_{l} \in$ $U$. We need not consider the case where $x_{k} \in U$, so we assume that $x_{k} \in S \backslash U$. As $x_{k} \in S \backslash U$ and $\operatorname{Dom}_{S}(U)=S$, by Result 1, we may let (2) be a zigzag of shortest possible length $m$ over $U$ with value $x_{k}$. Assume first that $1<k<l$. Then

$$
\begin{gathered}
x_{1}^{p} x_{2}^{p} \cdots x_{l}^{p}=x_{1}^{p} x_{2}^{p} \cdots a_{0}^{p} t_{1}^{p} x_{k+1}^{p} \cdots x_{l}^{p} \quad \text { (by Proposition } 4.6 \text { and equations (2)) } \\
=x_{1}^{p} x_{2}^{p} \cdots a_{0}^{p} b_{k+1}^{(1) p} \cdots b_{l}^{(1) p} t_{1}^{\prime}{ }^{p} z
\end{gathered}
$$

(by Result 4 and Proposition 4.6

$$
\begin{aligned}
& \text { for some } b_{k+1}^{(1)}, \ldots, b_{l}^{(1)} \in U \text {, and } t_{1}^{\prime} \in S \backslash U, \\
& \text { since } \left.t_{1} \in S \backslash U \text {, and where } z=x_{k+1}^{p} \cdots x_{l}^{p}\right)
\end{aligned}
$$$$
=x_{1}^{q} x_{2}^{q} \cdots a_{0}^{q} b_{k+1}^{(1) q} \cdots b_{l}^{(1) q} t_{1}^{\prime p} z \quad \text { (by the inductive hypothesis) }
$$$$
=w y_{1}^{q} a_{1}^{q} b_{k+1}^{(1) q} \cdots b_{l}^{(1) q} t_{1}^{\prime p} z \quad \text { (by Result } 6 \text { and equations (2), since } y_{1}, t_{1}^{\prime}
$$$$
\left.\in S \backslash U, \text { and where } w=x_{1}^{q} \cdots x_{k-1}^{q}\right)
$$$$
=w y_{1}^{\prime q} c_{1}^{(1) q} c_{2}^{(1) q} \cdots c_{k-1}^{(1) q} a_{1}^{q} b_{k+1}^{(1) q} \cdots b_{l}^{(1) q} t_{1}^{\prime p} z
$$

(by Result 4 for some $c_{1}^{(1)}, \ldots, c_{k-1}^{(1)} \in U$,

$$
\text { and } \left.y_{1}^{\prime} \in S \backslash U \text {, since } y_{1} \in S \backslash U\right)
$$$$
=w y_{1}^{\prime q} c_{1}^{(1) p} \cdots c_{k-1}^{(1) p} a_{1}^{p} b_{k+1}^{(1) p} \cdots b_{l}^{(1) p} t_{1}^{\prime p} z \quad \text { (since } U \text { satisfies (iii)) }
$$$$
=w y_{1}^{\prime q} c_{1}^{(1) p} \cdots c_{k-1}^{(1) p} a_{1}^{p} t_{1}^{p} z \quad\left(\text { since } t_{1}^{p}=b_{k+1}^{(1) p} \cdots b_{l}^{(1) p} t_{1}^{\prime p}\right)
$$

$$
\begin{aligned}
& =w y_{1}^{\prime q} c_{1}^{(1) p} \cdots c_{k-1}^{(1) p} a_{2}^{p} t_{2}^{p} z \quad \text { (by Result } 6 \text { and equations (2), } \\
& \text { since } \left.y_{1}^{\prime}, t_{1}, t_{2} \in S \backslash U\right) \\
& =w y_{m-1}^{\prime q} c_{1}^{(m-1) p} \cdots c_{k-1}^{(m-1) p} a_{2 m-2}^{p} t_{m}^{p} z \text { (for some } c_{1}^{(m-1)}, \ldots, c_{k-1}^{(m-1)} \in U \\
& \text { and } \left.y_{m-1}^{\prime} \in S \backslash U\right) \\
& =w y_{m-1}^{\prime q} c_{1}^{(m-1) p} \cdots c_{k-1}^{(m-1) p} a_{2 m-2}^{p} b_{k+1}^{(m) p} \cdots b_{l}^{(m)} t_{m}^{\prime p} z
\end{aligned}
$$

(by Result 4 and Proposition 4.6 for some $b_{k+1}^{(m)}, \ldots, b_{l}^{(m)} \in U$, and $t_{m}^{\prime} \in S \backslash U$, since $\left.t_{m} \in S \backslash U\right)$$$
=w y_{m-1}^{\prime q} c_{1}^{(m-1) q} \cdots c_{k-1}^{(m-1) q} a_{2 m-2}^{q} b_{k+1}^{(m) q} \cdots b_{l}^{(m) q_{t_{m}^{\prime}}^{\prime p} z}
$$

(since $U$ satisfies (iii))

$=w y_{m-1}^{q} a_{2 m-2}^{q} b_{k+1}^{(m) q} \cdots b_{l}^{(m) q_{t_{m}^{\prime}}{ }^{\prime} z} \quad\left(\right.$ since $\left.y_{m-1}^{q}=y_{m-1}^{\prime q} c_{1}^{(m-1) q} \cdots c_{k-1}^{(m-1) q}\right)$ 


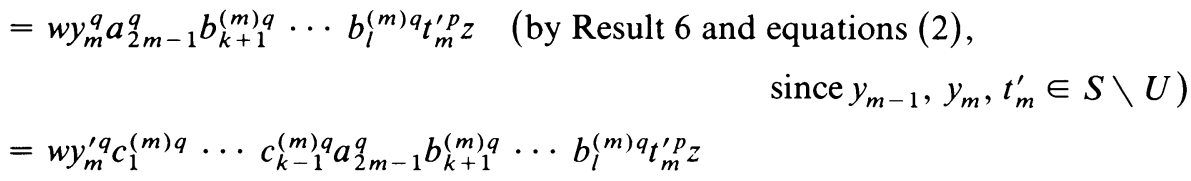

(by Result 4 and Proposition 4.6 for some $c_{1}^{(m)}, \ldots, c_{k-1}^{(m)} \in U$, and $y_{m}^{\prime} \in S \backslash U$, since $\left.y_{m} \in S \backslash U\right)$

$=w y_{m}^{\prime q} c_{1}^{(m) p} \cdots c_{k-1}^{(m) p} a_{2 m-1}^{p} b_{k+1}^{(m) p} \cdots b_{l}^{(m) p} t_{m}^{\prime p} z \quad$ (since $U$ satisfies (iii))

$=w y_{m}^{\prime q} c_{1}^{(m) p} \cdots c_{k-1}^{(m) p} a_{2 m-1}^{p} t_{m}^{p} z \quad\left(\right.$ since $\left.b_{k+1}^{(m) p} \cdots b_{l}^{(m) p} t_{m}^{\prime p}=t_{m}^{p}\right)$

$=w y_{m}^{\prime q} c_{1}^{(m) p} \cdots c_{k-1}^{(m) p} a_{2 m}^{p} x_{k+1}^{p} \cdots x_{l}^{p}$

(by Result 6 and equations (2),

$$
\text { since } \left.y_{m}^{\prime}, t_{m} \in S \backslash U, \text { and since } z=x_{k+1}^{p} \cdots x_{l}^{p}\right)
$$

$=w y_{m}^{\prime q} c_{1}^{(m) q} \cdots c_{k-1}^{(m) q} a_{2 m}^{q} x_{k+1}^{q} \cdots x_{l}^{q} \quad$ (since $U$ satisfies (iii))

$=w y_{m}^{q} a_{2 m}^{q} x_{k+1}^{q} \cdots x_{l}^{q} \quad\left(\right.$ since $\left.y_{m}^{\prime q} c_{1}^{(m) q} \cdots c_{k-1}^{(m) q}=y_{m}^{q}\right)$

$=x_{1}^{q} \cdots x_{k-1}^{q} x_{k}^{q} x_{k+1}^{q} \cdots x_{l}^{q} \quad$ (by Proposition 4.6 and equations (2)),

as required.

Finally, a proof in the remaining cases, namely when $k=1$ or $k=l$, can be obtained from the proof above by making the following conventions:

First when $k=1$,

(i) the word $w=1$,

(ii) the word $c_{1}^{(i) p} \cdots c_{k-1}^{(i) p}=c_{1}^{(i) q} \cdots c_{k-1}^{(i) q}=1$ and $y_{i}^{\prime}=y_{i}$ for $i=1,2, \ldots, m$.

Dually when $k=l$,

(i) the word $z=1$,

(ii) the word $b_{k+1}^{(i) p} \cdots b_{l}^{(i) p}=b_{k+1}^{(i) q} \cdots b_{l}^{(i) q}=1$ and $t_{m}^{\prime}=t_{m}$ for $i=1,2, \ldots, m$.

(iv) Assume $U$ satisfies (iv) and take any $x, y \in S$. First we consider the case where $x \in S \backslash U$ and $y \in U$ (the case where $x \in U$ and $y \in S \backslash U$ is symmetric to this case).

Since $x \in S \backslash U$, we may let (2), by Result 1, be a zigzag for $x$ of shortest possible length $m$ over $U$. Now

$$
\begin{aligned}
x^{p} y^{q} & =y_{m}^{p} a_{2 m}^{p} y^{q} \quad(\text { by Proposition } 4.6 \text { and equations }(2)) \\
& =y_{m}^{p} y^{r} a_{2 m}^{s} \quad(\text { since } U \text { satisfies (iv) }) \\
& =y_{m}^{p} y^{r}\left(a_{2 m-1} t_{m}\right)^{s} \quad(\text { from equations (2)) } \\
& =y_{m}^{p} y^{r} a_{2 m-1}^{s} t_{m}^{s} \quad\left(\text { by Result } 6, \text { since } y_{m}, t_{m} \in S \backslash U\right) \\
& =y_{m}^{p} a_{2 m-1}^{p} y^{q} t_{m}^{s} \quad(\text { since } U \text { satisfies (iv) }) \\
& =\left(y_{m} a_{2 m-1}\right)^{p} y^{q} t_{m}^{s} \quad\left(\text { by Result } 6, \text { since } y_{m}, t_{m} \in S \backslash U\right) \\
& =\left(y_{m-1} a_{2 m-2}\right)^{p} y^{q} t_{m}^{s} \quad(\text { from equations }(2)) \\
& =y_{m-1}^{p} a_{2 m-2}^{p} y^{q} t_{m}^{s} \quad\left(\text { by Result } 6, \text { since } y_{m-1}, t_{m} \in S \backslash U\right) \\
& =y_{m-1}^{p} y^{r} a_{2 m-2}^{s} t_{m}^{s} \quad(\text { since } U \text { satisfies (iv) })
\end{aligned}
$$




$$
\begin{aligned}
& =y_{m-1}^{p} y^{r}\left(a_{2 m-2} t_{m}\right)^{s} \quad\left(\text { by Result } 6, \text { since } y_{m-1}, t_{m} \in S \backslash U\right) \\
& =y_{m-1}^{p} y^{r}\left(a_{2 m-3} t_{m-1}\right)^{s} \quad(\text { from equations }(2)) \\
& \vdots \\
& =y_{1}^{p} y^{r}\left(a_{1} t_{1}\right)^{s} \\
& =y_{1}^{p} y^{r} a_{1}^{s} t_{1}^{s} \quad\left(\text { by Result } 6, \text { since } y_{1}, t_{1} \in S \backslash U\right) \\
& =y_{1}^{p} a_{1}^{p} y^{q} t_{1}^{s} \quad(\text { since } U \text { satisfies (iv) }) \\
& =\left(y_{1} a_{1}\right)^{p} y^{q} t_{1}^{s} \quad\left(\text { by Result } 6, \text { since } y_{1}, t_{1} \in S \backslash U\right) \\
& =a_{0}^{p} y^{q} t_{1}^{s} \quad(\text { from equations }(2)) \\
& =y^{r} a_{0}^{s} t_{1}^{s} \quad(\text { since } U \text { satisfies (iv)) } \\
& =y^{r} x^{s} \quad(\text { by Proposition } 4.6 \text { and equations }(2)),
\end{aligned}
$$

as required.

So we assume next that $x, y \in S \backslash U$. By Result 1, we may let (2) be a zigzag for $x$ of shortest possible length $m$ over $U$. Then

$$
\begin{aligned}
& x^{p} y^{q}=y_{m}^{p} a_{2 m}^{p} y^{q} \quad \text { (by Proposition } 4.6 \text { and equations (2)) } \\
& =y_{m}^{p} y^{r} a_{2 m}^{s} \text { (by the first part of the proof) } \\
& =y_{m}^{p} y^{r}\left(a_{2 m-1} t_{m}\right)^{s} \quad \text { (from equations (2)) } \\
& =y_{m}^{p} y^{r} a_{2 m-1}^{s} t_{m}^{s} \quad \text { (by Result 6, since } y_{m}, t_{m} \in S \backslash U \text { ) } \\
& =y_{m}^{p} a_{2 m-1}^{p} y^{q} t_{m}^{s} \quad \text { (by the first part of the proof) } \\
& \left.=\left(y_{m} a_{2 m-1}\right)^{p} y^{q} t_{m}^{s} \quad \text { (by Result 6, since } y_{m}, t_{m} \in S \backslash U\right) \\
& =\left(y_{m-1} a_{2 m-2}\right)^{p} y^{q} t_{m}^{s} \quad \text { (from equations (2)) } \\
& \left.=y_{m-1}^{p} a_{2 m-2}^{p} y^{q} t_{m}^{s} \quad \text { (by Result 6, since } y_{m-1}, t_{m} \in S \backslash U\right) \\
& =y_{m-1}^{p} y^{r} a_{2 m-2}^{s} t_{m}^{s} \quad \text { (by the first part of the proof) } \\
& =y_{m-1}^{p} y^{r}\left(a_{2 m-2} t_{m}\right)^{s} \quad\left(\text { by Result 6, since } y_{m-1}, t_{m} \in S \backslash U\right) \\
& =y_{m-1}^{p} y^{r}\left(a_{2 m-3} t_{m-1}\right)^{s} \quad \text { (from equations (2)) } \\
& \text { : } \\
& =y_{1}^{p} y^{r}\left(a_{1} t_{1}\right)^{s} \\
& \left.=y_{1}^{p} y^{r} a_{1}^{s} t_{1}^{s} \quad \text { (by Result 6, since } y_{1}, t_{1} \in S \backslash U\right) \\
& =y_{1}^{p} a_{1}^{p} y^{q} t_{1}^{s} \quad \text { (by the first part of the proof) } \\
& \left.=\left(y_{1} a_{1}\right)^{p} y^{q} t_{1}^{s} \quad \text { (by Result 6, since } y_{1}, t_{1} \in S \backslash U\right) \\
& =a_{0}^{p} y^{q} t_{1}^{s} \quad \text { (from equations (2)) } \\
& =y^{r} a_{0}^{s} t_{1}^{s} \quad \text { (by the first part of the proof) } \\
& =y^{r} x^{s} \quad \text { (by Proposition } 4.6 \text { and equations (2)), }
\end{aligned}
$$

as required. This completes the proof of part (iv). 
(v) Assume $U$ satisfies $x^{p}=0$ and take any $x, y \in S$; we show that $x^{p} y=y x^{p}=$ $x^{p}$.

Case (a). $x \in S \backslash U, y \in U$. Let (2), by Result 1, be a zigzag for $x$ over $U$ of shortest possible length $m$. Then

$$
\begin{aligned}
x^{p} y & =y_{m}^{p} a_{2 m}^{p} y \quad(\text { by Proposition } 4.6 \text { and equations }(2)) \\
& =y_{m}^{p} a_{2 m}^{p} \quad(\text { since } U \text { satisfies }(\mathrm{v})) \\
& =x^{p} .
\end{aligned}
$$

Similarly $y x^{p}=x^{p}$, as required.

Case (b). $x \in U, y \in S \backslash U$. Since $y \in S \backslash U$, we may let (2), by Result 1 , be a zigzag of length $m$ over $U$ with value $y$. Then

$$
\begin{aligned}
x^{p} y & =x^{p} a_{0} t_{1}=x^{p} a_{1} t_{1} \quad(\text { since } U \text { satisfies }(\mathrm{v})) \\
& =x^{p} a_{2} t_{2} \quad(\text { from equations }(2)) \\
& =x^{p} a_{3} t_{2} \quad(\text { since } U \text { satisfies }(\mathrm{v})) \\
& \vdots \\
& =x^{p} a_{2 m-1} t_{m} \\
& =x^{p} a_{2 m}=x^{p} \quad(\text { since } U \text { satisfies }(\mathrm{v})) .
\end{aligned}
$$

Similarly $y x^{p}=x^{p}$, as required.

Case (c). $x, y \in S \backslash U$. By Result 1, let (2) be a zigzag for $x$ of shortest possible length $m$ over $U$. Then

$$
\begin{aligned}
x^{p} y & =y_{m}^{p} a_{2 m}^{p} y \quad(\text { by Proposition } 4.6 \text { and equations }(2)) \\
& =y_{m}^{p} a_{2 m}^{p} \quad(\text { from case }(\mathrm{b}) \text { above }) \\
& =x^{p} .
\end{aligned}
$$

Similarly $y x^{p}=x^{p}$, are required.

(vi) Assume $U$ satisfies (vi) and take any $x, y, z \in S$; we prove that $x^{p} y^{q} z=$ $z x^{p} y^{q}=x^{p} y^{q}$.

Case (a). $x, y \in U, z \in S \backslash U$. Let (2), by Result 1, be a zigzag of shortest possible length $m$ over $U$ with value $z$. Then

$$
\begin{aligned}
x^{p} y^{q} z & =x^{p} y^{q} a_{0} t_{1} \quad(\text { from equations (2)) } \\
& =x^{p} y^{q} a_{1} t_{1} \quad(\text { since } U \text { satisfies (vi)) } \\
& =x^{p} y^{q} a_{2} t_{2} \quad(\text { from equations (2)) } \\
& \vdots \\
& =x^{k} y^{q} a_{2 m-2} t_{m} \\
& =x^{p} y^{q} a_{2 m-1} t_{m} \quad(\text { since } U \text { satisfies (vi)) } \\
& =x^{p} y^{q} a_{2 m} \quad(\text { from equations (2)) } \\
& =x^{p} y^{q} \quad(\text { since } U \text { satisfies (vi)). }
\end{aligned}
$$

By a similar argument we can show easily that $z x^{p} y^{q}=x^{p} y^{q}$. Therefore $x^{p} y^{q} z=$ $z x^{p} y^{q}=x^{p} y^{q}$, as required. 
Case (b). $y, z \in U, x \in S \backslash U$. As $x \in S \backslash U$, by Result 1, we may let (2) be a zigzag of shortest possible length $m$ over $U$ with value $x$. Then

$$
\begin{aligned}
x^{p} y^{q} z & \left.=y_{m}^{p} a_{2 m}^{p} y^{q} z \quad \text { (by Proposition } 4.6 \text { and equations }(2)\right) \\
& =y_{m}^{p} a_{2 m}^{p} y^{q} \quad(\text { since } U \text { satisfies (vi)) } \\
& =x^{p} y^{q} \quad(\text { by Proposition } 4.6 \text { and equations }(2)) .
\end{aligned}
$$

Also

$$
\begin{aligned}
z x^{p} y^{q} & =z y_{m}^{p} a_{2 m}^{p} y^{q} \quad(\text { by Proposition } 4.6 \text { and equations }(2)) \\
& =y_{m}^{p} a_{2 m}^{p} y^{q} \quad(\text { from Case }(\mathrm{a})) \\
& =x^{p} y^{q} \quad(\text { by Proposition } 4.6 \text { and equations (2)) }
\end{aligned}
$$

Therefore $x^{p} y^{q} z=z x^{p} y^{q}=x^{p} y^{q}$, as required.

Case (c). $x, z \in U, y \in S \backslash U$. This case is dual to Case (b).

Case (d). $z \in U, x, y \in S \backslash U$. Let (2), by Result 1, be a zigzag of shortest possible length $m$ over $U$ with value $x$. Now

$$
\begin{aligned}
x^{p} y^{q} z & =y_{m}^{p} a_{2 m}^{p} y^{q} z \quad(\text { by Proposition } 4.6 \text { and equations (2)) } \\
& =y_{m}^{p} a_{2 m}^{p} y^{q} \quad(\text { from Case }(\mathrm{c})) \\
& =x^{p} y^{q} \quad(\text { by Proposition } 4.6 \text { and equations (2)) }
\end{aligned}
$$

Since $y \in S \backslash U$, by Result 1 , we may let $y=b_{0} z_{1}=s_{1} b_{1} z_{1}$ be the first two lines of a zigzag for $y$ with $b_{0}, b_{1} \in U$, and $s_{1}, z_{1} \in S \backslash U$. Now

$$
\begin{aligned}
z x^{p} y^{q} & =z y_{m}^{p} a_{2 m}^{p} b_{0}^{q} z_{1}^{q} \quad \text { (by Proposition } 4.6 \text { and equations (2)) } \\
& =y_{m}^{p} a_{2 m}^{p} b_{0}^{q} z_{1}^{q} \quad(\text { from Case }(\mathrm{a})) \\
& =x^{p} y^{q} \quad(\text { by Proposition } 4.6 \text { and equations }(2)) .
\end{aligned}
$$

Therefore $x^{p} y^{q} z=z x^{p} y^{q}=x^{p} y^{q}$, as required.

Case (e). $y \in U, x, z \in S \backslash U$ or $x \in U, y, z \in S \backslash U$ or $x, y, z \in S \backslash U$. As $z \in S \backslash U$, by Result 1, we may let (2) be a zigzag of length $m$ over $U$ with value $z$. Now

$$
\begin{aligned}
x^{p} y^{q} z & =x^{p} y^{q} a_{0} t_{1} \quad(\text { from equations (2)) } \\
& =x^{p} y^{q} a_{1} t_{1} \quad(\text { from Cases (b) },(\mathrm{c}) \text { and }(\mathrm{d})) \\
& =x^{p} y^{q} a_{2} t_{2} \quad(\text { from equations }(2)) \\
& \vdots \\
& =x^{p} y^{q} a_{2 m-2} t_{m} \\
& =x^{p} y^{q} a_{2 m-1} t_{m} \quad(\text { from Cases }(\mathrm{b}),(\mathrm{c}) \text { and }(\mathrm{d})) \\
& =x^{p} y^{q} a_{2 m} \quad(\text { from equations }(2)) \\
& =x^{p} y^{q} \quad(\text { from Case }(\mathrm{d})) .
\end{aligned}
$$

The dual argument shows that $z x^{p} y^{q}=x^{p} y^{q}$. Therefore

$$
x^{p} y^{q} z=z x^{p} y^{q}=x^{p} y^{q}
$$

as required, thus completing the proof of Theorem 4.7. 
ACKNOWLedgement. I thank Tom Hall, my supervisor, for his help and encouragement throughout the writing of this paper.

\section{REFERENCES}

1. A. Bulazewska and J. Krempka, On epimorphisms in the category of all associative rings, Bull. Acad. Polon. Sci. Sér. Sci. Math. Astronom. Phys. 23 (1975).

2. W. Burgess, The meaning of mono and epi in some familiar categories, Canad. Math. Bull. 8 (1965).

3. A. H. Clifford and G. B. Preston, The algebraic theory of semigroups, Vol. 1, Math. Surveys, No. 7, Amer. Math. Soc., Providence, R. I., 1961: Vol. II, 1967.

4. B. J. Gardner, $A$ note on ring epimorphisms and polynomial identities, Comment. Math. Univ. Carolin. 20 (1979).

5. P. M. Higgins, Saturated and epimorphically closed varieties of semigroups, J. Austral. Math. Soc. Ser. A 36 (1984), 153-175.

6. E E Eimorphisms, permutation identities and finite semigroups, Semigroup Forum (to appear).

7. J. M. Howie, An introduction to semigroup theory, London Math. Soc. Monographs, vol. 7, Academic Press, 1976.

8. J. M. Howie and J. R. Isbell, Epimorphisms and dominions. II, J. Algebra 6 (1967), 7-21.

9. J. R. Isbell, Epimorphisms and dominions, Proc. Conf. on Categorical Algebra (La Jolla, 1965), Lange \& Springer, Berlin, 1966, pp. 232-246.

10. N. M. Khan, Epimorphisms, dominions and varieties of semigroups, Semigroup Forum 25 (1982), 331-337.

11. On saturated varieties and consequences of permutation identities. J. Austral. Math. Soc. (to appear).

Department of Mathematics, Monash University, Clayton, Victoria, Australia 3168

Current address: Department of Mathematics, Aligarh Muslim University, Aligarh, India 202001 